\section{ERROS DE PRESCRIÇÃO DE MEDICAMENTOS: UMA AVALIAÇÃO DA PRESCRIÇÃO NA PEDIATRIA DE UM HOSPITAL ESCOLA}

Dayane Beserra Costa ${ }^{1}$

Luciana Lucena Aranha de

Macedo $^{2}$

Rosa Águida Donosora de Melo

Souto ${ }^{1}$

Ana Luiza dos Santos ${ }^{2}$

\title{
RESUMO
}

Introdução: Os erros de medicação são eventos adversos comuns e podem assumir relevantes repercussões econômicas e sociais. Os erros de prescrição contribuem de maneira significativa para o índice total de erros de medicação e têm alto potencial para resultarem em consequências prejudiciais para o usuário. Objetivo: A pesquisa objetivou analisar a qualidade das prescrições em pediatria com ênfase nos erros de prescrição. Métodos: Trata-se de um estudo observacional, descritivo, transversal com delineamento retrospectivo. Foi realizado na Clínica Pediátrica de um hospital de ensino. As variáveis em estudo seguiram o que preconiza o Protocolo de Segurança na Prescrição, Uso e Administração de Medicamentos do Ministério da Saúde. Os dados foram armazenados, organizados e tratados estatisticamente com auxílio do programa SPSS versão 20. Resultados: Foram analisadas 898 prescrições, contendo um total de 3858 itens, e estavam ausentes nas prescrições as seguintes variáveis analisadas: nome completo do usuário $2,7 \%$, número do prontuário $61,5 \%$, ala $1,2 \%$, leito $80,5 \%$, enfermaria $15,9 \%$. A forma farmacêutica esteve ausente em $26,1 \%$, a concentração do fármaco não foi especificada em $20,3 \%$ e o tempo de tratamento não foi evidenciado em $85,2 \%$ das prescrições avaliadas. O volume e o tipo do diluente não foram descritos em 12,8\% e 7,4\% respectivamente, assim como não foram especificadas nas prescrições em estudo a velocidade e o tempo de infusão em 96,6\% e 79,3\% respectivamente. Conclusões: A pesquisa realizada revela que a qualidade da prescrição do hospital em questão precisa de ajustes importantes em vários aspectos, principalmente no que diz respeito aos medicamentos.

Palavras-chave: Segurança do paciente, erros de medicação, pediatria.

\section{INTRODUÇÃO}

Os eventos adversos (EA) relacionados a medicamentos podem acarretar significativos agravos à saúde dos pacientes, sendo considerados atualmente um importante problema de saúde pública. Dentre esses eventos adversos, os erros de medicação são ocorrências comuns e podem assumir relevantes repercussões econômicas e sociais ${ }^{1}$.

Os erros de medicação são qualquer evento evitável que pode levar ao uso inadequado de medicamentos, independente do risco de lesar ou não o paciente, e do fato de o medicamento se encontrar sob a posse de profissionais de saúde, do paciente ou do consumidor ${ }^{2}$.

As causas desses erros podem estar relacionadas a qualquer ponto no sistema de medicação, no entanto, não é raro acontecer algum erro de medicação e apenas um profissional ser responsabilizado, o que corrobora para o baixo índice de notificações ${ }^{3}$.

Dean et al. ${ }^{4}$, definem erro de prescrição como um erro de decisão, não intencional, que pode reduzir a probabilidade do tratamento ser efetivo ou aumentar o risco de lesão no paciente. Posteriormente, Cassiani ${ }^{5}$ traz novos elementos para a definição de erros de prescrição e os define como erros relacionados à seleção incorreta do medicamento, de acordo com contraindicações, alergias conhecidas, e entre outros fatores, monitoração, dose, via de administração, velocidade de infusão, concentração, instruções de uso inadequadas ou insuficientes, ilegibilidade da prescrição ou ordens que possam induzir ao erro.

Os erros devido à prescrição contribuem de maneira significativa para o índice total de erros de medicação e têm alto potencial para resultarem em consequências prejudiciais para o usuário ${ }^{6}$. Pesquisas demonstram que a cada dez pacientes admitidos em uma unidade hospitalar um está em risco potencial ou efetivo de erro de medicação ${ }^{7}$.

É de conhecimento dos profissionais de saúde que prescrições ambíguas, ilegíveis, de difícil compreensão ou incompletas, bem como a falta de padronização da nomenclatura de medicamentos prescritos (nome comercial ou genérico), o uso de abreviaturas, uso de expressões vagas e a presença de rasuras são fatores que contribuem diretamente para a ocorrência desses eventos ${ }^{8}$.

Com a implantação do Programa Nacional Para a Segurança do Paciente, algumas estratégias foram traçadas para tentar minimizar a ocorrência de eventos adversos relacionados a medicamentos, dentre estas estratégias estão a elaboração de

\section{Hospital Universitário Lauro Wanderley \\ 2 Universidade Federal da Paraíba}

Recebido: $07 / 03 / 2018$

Aceito: $30 / 06 / 2018$

Como citar este artigo: Costa DB, Macedo LLA, Souto RADM e Santos AL. Erros de prescrição de medicamentos: uma avaliação da prescrição na pediatria de um hospital escola.

Rev. Bras. Farm. Hosp. Serv. Saúde, 9(2): 01-05, 2019.

Doi: 10.30968/rbfhss.2018.092.002

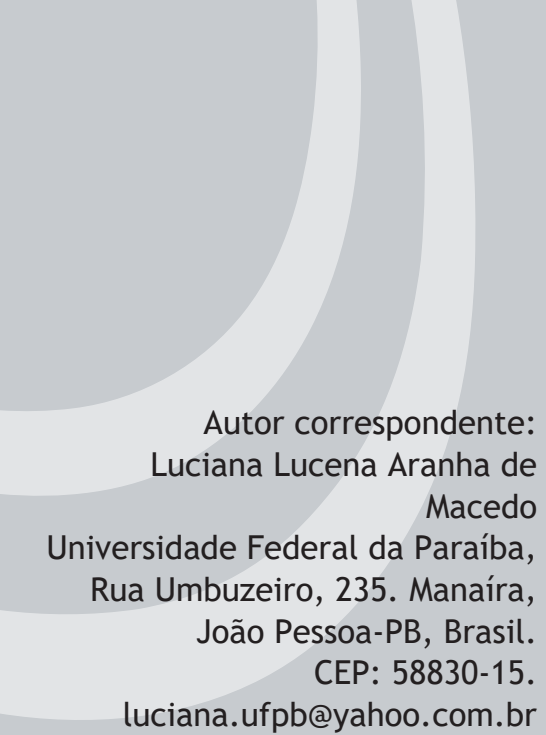


protocolos com temas relevantes para a segurança do paciente, desta forma, um dos protocolos elaborados pelo Ministério da Saúde em parceria com a vigilância sanitária e a Fundação Oswaldo Cruz foi o protocolo de segurança na prescrição, uso e administração de medicamentos que traz alguns pontos que devem ser observados e seguidos para que se tenha uma prescrição considerada segura ${ }^{9}$.

Essa pesquisa objetivou analisar a qualidade das prescrições em pediatria com ênfase nos erros de prescrição, para com isso, contribuir com a redução de erros de medicação e promover o uso racional de medicamentos, proporcionando, desta forma, maior eficácia e segurança ao paciente em seu tratamento farmacológico.

\section{MÉTODOS}

Trata-se de um estudo observacional, de natureza descritiva, transversal com delineamento retrospectivo. Foi realizado na clínica pediátrica do Hospital Universitário Lauro Wanderley (HULW) em João Pessoa - PB, a abordagem de avaliação da qualidade das prescrições se deu por meio da avaliação das prescrições dos usuários internos no referido setor. O hospital no qual foi realizado o estudo possui uma unidade de internação pediátrica em pleno funcionamento, conta com vinte e dois leitos e é porta de entrada para internações advindas de todo o estado da Paraíba. Os dados foram coletados das segundas vias das prescrições de crianças internas na clínica pediátrica no período de maio e junho de 2015.

A amostra em estudo foi composta por prescrições de crianças e adolescentes, admitidos na unidade de internação pediátrica desta unidade hospitalar durante o período de vigência do estudo. Os critérios de inclusão para a análise das prescrições foram: ter no mínimo um medicamento prescrito e mais de vinte e quatro horas de permanência no setor.

As variáveis em estudo seguiram o que preconiza o protocolo de segurança na prescrição, uso e administração de medicamentos do Ministério da Saúde ${ }^{9}$, desta forma, foram avaliados os seguintes pontos: identificação do paciente, identificação do prescritor, identificação da instituição, identificação da data na prescrição, legibilidade, tipo de prescrição utilizada, expressão adequada de doses, sinalização de alergias medicamentosas, especificação da duração do tratamento, especificação na prescrição da posologia, diluição, velocidade, tempo de infusão, via de administração e a utilização de expressões vagas.

Os termos considerados pelo protocolo de segurança na prescrição, uso e administração de medicamentos do Ministério da Saúde ${ }^{9}$ como expressões vagas são: "se necessário", "a critério médico", "usar como habitual", "uso contínuo", "não parar” e "usar como de costume", a utilização dessas expressões predispõe a administração do fármaco sem julgamento criterioso o que corrobora diretamente para o acontecimento de incidentes ${ }^{10}$.

Devido à grande subjetividade que envolve a variável legibilidade optou-se por estabelecer padrões de avaliação, desta forma, reduzindo aspectos da subjetividade envolvida no julgamento. A variável foi classificada em duas possibilidades: a) Grafia com boa legibilidade: lida normalmente sem problemas para entendimento da escrita; e b) Grafia ilegível: impossível o entendimento da escrita ${ }^{11}$.

Os dados foram armazenados, organizados e tratados estatisticamente com auxílio do programa SPSS versão 20, foi realizada uma análise descritiva de todas as variáveis incluídas no estudo, distribuições de frequência e estatística descritiva das variáveis quantitativas.

Vale ressaltar que para o desenvolvimento desta pesquisa foram respeitados todos os princípios éticos que estão previstos na nova Resolução no. 466 de 12 de dezembro de 2012 do Conselho Nacional de Saúde (CNS) que aprova as diretrizes e normas regulamentadoras de pesquisas que envolvem seres humanos. Foi garantido aos sujeitos pesquisados total anonimato, primando-se ainda pelo livre acesso aos resultados da pesquisa, conforme estabelece a supracitada Resolução. A referida pesquisa foi aprovada pelo Comitê de Ética em Pesquisa do Hospital Universitário Lauro Wanderley com o seguinte número de protocolo 1.138 .356$.

\section{RESULTADOS}

Foram analisadas 898 prescrições, contendo um total de 3858 itens, os quais foram observados individualmente. A média de medicamentos por prescrição foi de 4,2 medicamentos.

As características demográficas e clínicas são mostradas na Tabela 1, que traz dados referentes ao sexo, idade, diagnóstico e medicamentos prescritos para os usuários internos na clínica pediátrica. Em relação ao sexo, não há praticamente diferença entre os usuários.

Os usuários tinham idades compreendidas entre 0 e 18 anos e, vale a pena ressaltar que $12,8 \%$ das prescrições não havia a especificação da idade do usuário, dado extremamente importante para escolha e cálculo da dose do medicamento. Foram encontrados 69 diagnósticos diferentes. Foram prescritos 102 diferentes princípios ativos, que estão descritos na Tabela 1.

Tabela 1 : Distribuição das prescrições quanto aos dados demográficos e clínicos

\begin{tabular}{|c|c|c|}
\hline Variável & Frequência & $\begin{array}{l}\text { Porcentagem } \\
\quad(n=898)\end{array}$ \\
\hline \multicolumn{3}{|l|}{ Idade (anos) } \\
\hline $0-4$ & 465 & $51,8 \%$ \\
\hline $5-9$ & 115 & $12,8 \%$ \\
\hline $10-14$ & 169 & $18,8 \%$ \\
\hline $15-18$ & 34 & $3,8 \%$ \\
\hline Não informado & 115 & $12,8 \%$ \\
\hline \multicolumn{3}{|l|}{ Sexo } \\
\hline Feminino & 429 & $47,8 \%$ \\
\hline Masculino & 449 & $50,2 \%$ \\
\hline Não informado & 20 & $2,2 \%$ \\
\hline \multicolumn{3}{|l|}{ Diagnóstico } \\
\hline Pneumonia & 129 & $14,4 \%$ \\
\hline Lúpus Eritematoso Sistêmico & 58 & $6,5 \%$ \\
\hline Infecção do Trato Urinário & 50 & $5,6 \%$ \\
\hline Bronquiolite & 31 & $3,5 \%$ \\
\hline Leucemia & 29 & $3,2 \%$ \\
\hline Não especificado & 87 & $9,7 \%$ \\
\hline Outros & 514 & $57,1 \%$ \\
\hline \multicolumn{3}{|l|}{ Medicamentos Prescritos } \\
\hline Dipirona & 615 & $15,9 \%$ \\
\hline Simeticona & 260 & $6,7 \%$ \\
\hline Omeprazol & 253 & $6,6 \%$ \\
\hline Paracetamol & 213 & $5,5 \%$ \\
\hline Ceftriaxona & 128 & $3,3 \%$ \\
\hline Ondansetrona & 126 & $3,3 \%$ \\
\hline Hidrocortisona & 111 & $2,9 \%$ \\
\hline Domperidona & 104 & $2,7 \%$ \\
\hline Furosemida & 97 & $2,5 \%$ \\
\hline Prednisona & 80 & $2,1 \%$ \\
\hline Espironolactona & 78 & $2,0 \%$ \\
\hline Outros medicamentos & 1793 & $46,5 \%$ \\
\hline
\end{tabular}

O Gráfico 1 traz informações necessárias para uma prescrição segura, segundo o protocolo de segurança na prescrição, uso e 
administração de medicamentos do Ministério da Saúde ${ }^{9}$, foi observado que o número do prontuário não foi encontrado em $61,5 \%$, e em $80,5 \%$ não foi mencionado o leito em que o paciente se encontrava interno, o nome da enfermaria não constava em 15,9\% das prescrições avaliadas.

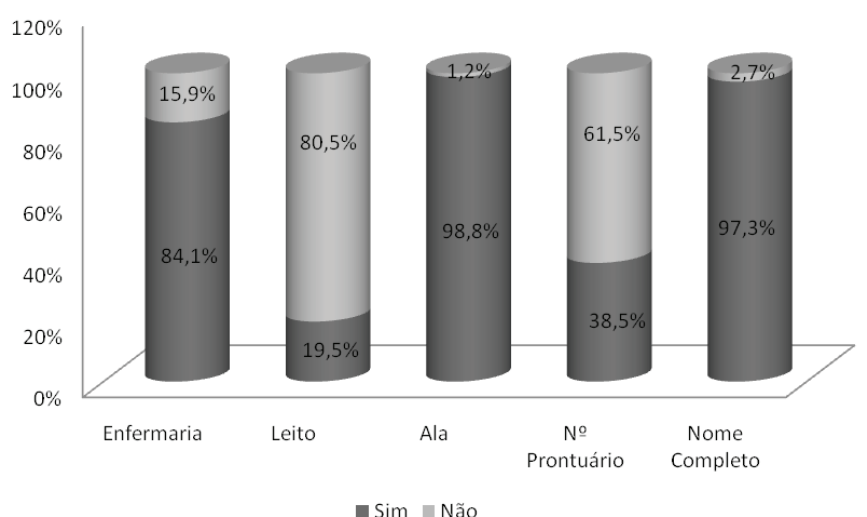

Gráfico 1: Distribuição das prescrições quanto a presença de dados referentes à identificação do paciente.

Em relação à identificação do prescritor, em 4,7\% não havia o nome completo do prescritor responsável pela prescrição e em 3,6\% não havia o número do registro no conselho profissional. A data da prescrição não estava especificada em 1,4\% dos documentos avaliados. A sinalização de alergias medicamentosas na prescrição foi encontrada em $0,4 \%$ das prescrições em estudo.

O nome do estabelecimento estava presente em 100\% das prescrições, no entanto, o endereço e telefone do estabelecimento de saúde não fazem parte da prescrição padrão do hospital em estudo, desta forma, 100\% das prescrições avaliadas apresentaram ausência desses dados.

Em relação à legibilidade $98,8 \%$ das prescrições foram classificadas como legíveis, no entanto, em 1,4\% não foi possível identificar os itens prescritos, configurando, desta forma, ilegibilidade. Quanto ao tipo de prescrição $85,3 \%$ eram prescrições do tipo digitada, $15 \%$ carbonadas e $0,3 \%$ prescrições feitas à mão sem o uso do carbono para gerar a segunda via, como pode ser observado no Gráfico 2.

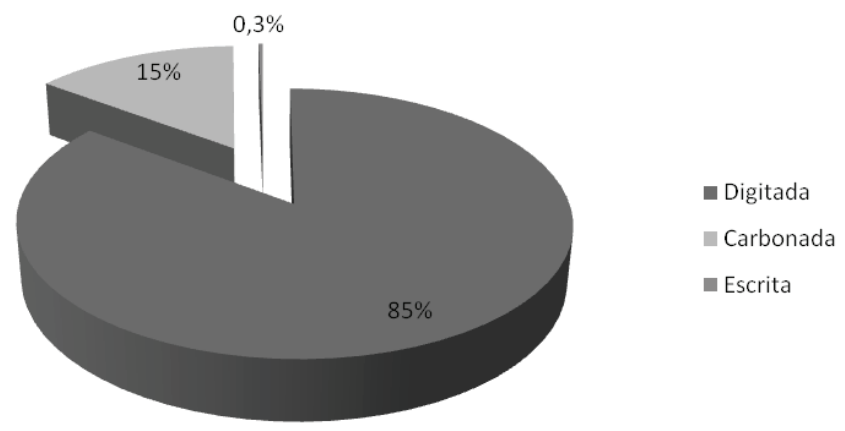

Gráfico 2: Distribuição das prescrições segundo o tipo de prescrição

No que diz respeito às informações essenciais relacionadas ao medicamento como: forma farmacêutica, concentração da droga, intervalo entre as doses, tempo de tratamento, tipo e volume do diluente, velocidade e tempo de infusão e via de administração, os gráficos 3 e 4 apresentam os principais dados obtidos na pesquisa.

A forma farmacêutica esteve ausente em $26,1 \%$, a concentração do fármaco não foi especificada em $20,3 \%$, o tempo de tratamento não foi evidenciado em $85,2 \%$ das prescrições avaliadas. $O$ volume e o tipo do diluente não foram descritos em $12,8 \%$ e 7,4\% respectivamente, assim como também não foram especificadas nas prescrições em estudo a velocidade e o tempo de infusão em $96,6 \%$ e $79,3 \%$ respectivamente.

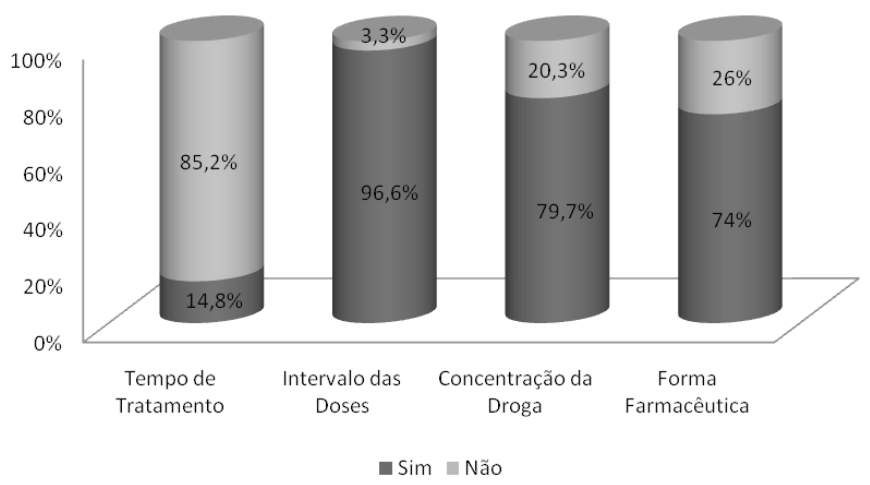

Gráfico 3: Distribuição das prescrições quanto à presença de informações essenciais relacionadas aos medicamentos.

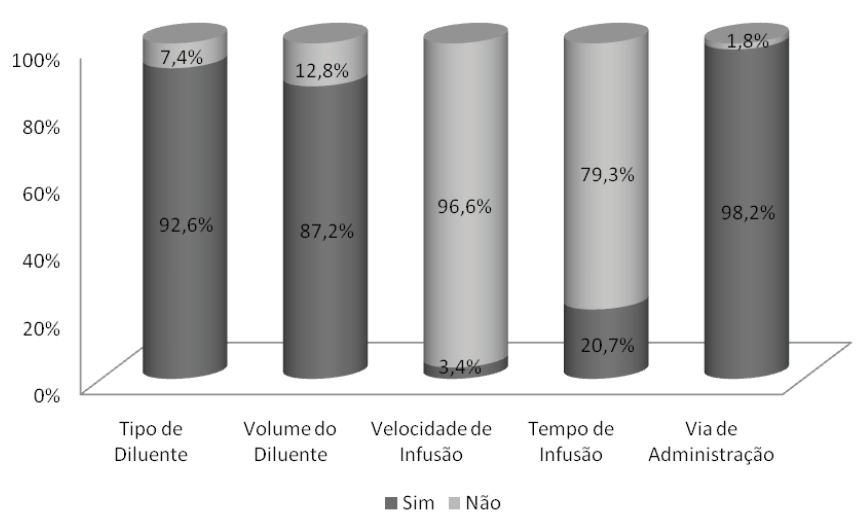

Gráfico 4: Distribuição das prescrições quanto à presença de informações essenciais relacionadas aos medicamentos e sua via de administração.

Quanto à presença de abreviaturas, foi observado que 96,2\% das prescrições apresentavam algum tipo de abreviatura, ocorrendo com maior frequência as abreviaturas VO em 51,1\% e EV em 38\%. O uso de expressões vagas, tais como "usar como de costume", "a critério médico" "usar se necessário" sem a correta explicação por escrito de como deve ser realizada a terapia medicamentosa, foi evidenciada em $24,4 \%$ das prescrições, desse total 91,5\% eram expressões do tipo "usar se necessário" expressas nas prescrições por meio da abreviatura SN, 8,5\% "usar a critério médico" expressas através da abreviatura ACM, como pode ser observado no Gráfico 5.

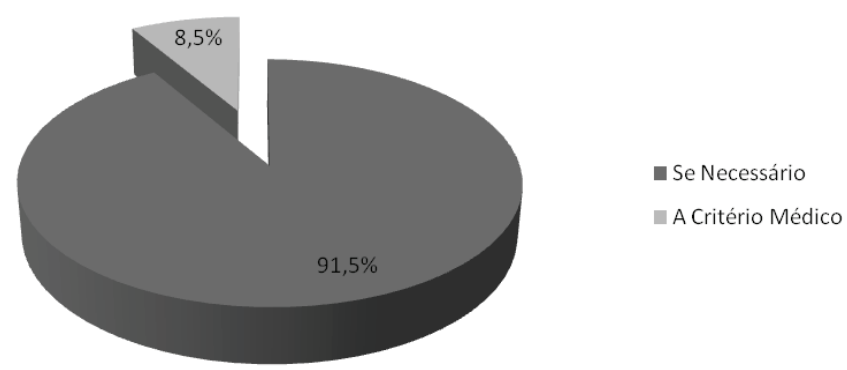

Gráfico 5: Distribuição das prescrições quanto ao tipo de expressão vaga encontrada. 


\section{DISCUSSÃO}

Alguns estudos como o de Uchôa e Araújo ${ }^{10}$, avaliaram a qualidade das prescrições e encontraram uma média de medicamentos prescritos por paciente igual a 4,47, compatível com o valor encontrado nessa pesquisa, no entanto, a Organização Mundial de Saúde (OMS) ${ }^{12}$ recomenda que sejam prescritos dois medicamentos por prescrição, sendo assim, os dados encontrados nos estudos citados estão bem acima do que é preconizado pela OMS para que seja configurado o uso racional de medicamentos. Além do número de medicamentos prescritos para que seja configurado o uso racional de medicamentos deve-se levar em consideração a prescrição adequada, a disponibilidade do medicamento a preços acessíveis, a dispensação em condições adequadas, o conjunto de doses indicadas, nos intervalos definidos e no período de tempo indicado de medicamentos eficazes, seguros e de qualidade ${ }^{13}$.

A identificação do paciente consiste num processo pelo qual se assegura ao paciente que a ele é destinado algum tipo específico de procedimento ou tratamento medicamentoso, prevenindo a ocorrência de erros e enganos que possam levar a uma lesão. Os erros de identificação podem ocorrer desde a admissão do usuário até a alta, ou seja, em todas as fases do diagnóstico e tratamento, desta forma o protocolo de identificação do paciente, do Ministério da Saúde preconiza que para que haja a identificação segura do paciente é necessário utilizar no mínimo dois identificadores, dentre eles destaca-se o nome completo do usuário e o número do prontuário?

Os problemas relacionados à identificação do paciente configuram uma grave situação que pode aumentar a probabilidade de erros, dados inferiores foram encontrados quando comparados com um estudo realizado por Rosa et al ${ }^{11}$, no qual foi observado que $47 \%$ das prescrições apresentavam alguma ausência de informação na identificação do paciente e em 33,7\% na identificação do prescritor, Bózoli14 observou que em $34,5 \%$ das prescrições não havia a especificação do responsável pela prescrição, Araújo e Uchôa ${ }^{10}$ mencionam em seu estudo que $80 \%$ das prescrições não apresentavam carimbo do médico prescritor e em 41,07\% não havia o número do registro profissional.

A prescrição é um documento legal, e como tal, deve obrigatoriamente conter a identificação não apenas do paciente, mas também do emitente, para que em situações de dúvidas, seja possível a localização do responsável pela prescrição ${ }^{15}$. Além do nome do paciente outras ferramentas podem ser utilizadas para minimizar erros de identificação, como o número do prontuário, no entanto, Araújo e Uchôa ${ }^{10}$ encontraram a ausência dessa informação em $41 \%$ das prescrições dado equivalente ao estudo aqui descrito. Em um ambiente hospitalar, onde há centenas de pacientes internos omitir esse tipo de informação pode levar a ocorrência de graves incidentes ${ }^{11}$.

Quanto a legibilidade, foi encontrado um percentual bem inferior aos descritos nos estudos de Silva et al. ${ }^{16}$, onde $28,92 \%$ das prescrições foram consideradas ilegíveis, Leite e Silvério ${ }^{17}$ encontraram $36 \%$ de ilegibilidade nas prescrições avaliadas. $\mathrm{O}$ baixo percentual de prescrições ilegíveis pode ser explicado devido ao fato de a grande maioria das prescrições avaliadas nessa pesquisa serem do tipo digitada, minimizando, desta forma, as chances de não serem entendidas ou serem mal interpretadas.

Ao compararmos os dados dessa pesquisa com a literatura observamos que os resultados (Gráfico 3) foram próximos aos encontrados por Rosa et al., onde não foram mencionadas nas prescrições informações referentes a forma farmacêutica em $84 \%$, concentração $62 \%$, dose $17 \%$, intervalo entre doses $12 \%$ e via de adminitração em $13 \%$ das prescrições ${ }^{11}$, Aldrigue et. al., encontraram percentuais inferiores, onde houve ausência da forma farmacêutica em $0,5 \%$, da concentração em $20 \%$, da dose $0,5 \%$, do intervalo entre doses $1 \%$ e da duração do tratamento em $55 \%$ das prescrições avaliadas ${ }^{18}$. Silvério e Leite ${ }^{17}$ afirmam que houve ausência de informações referentes a forma farmacêutica em $64 \%$, de concentração em $47 \%$, de dose em $22 \%$, do intervalo entre as doses em 63\%, da duração do tratamento em 30\% e a via de administração estava ausente em $84 \%$ das prescrições, os dados de Silvério e Leite ${ }^{17}$ foram os que mais se aproximaram quantitativamente do estudo em questão. Aspden et al., afirmam em seu estudo que para prevenir esses tipos de erros em ambientes hospitalares as recomendações com maior evidência científica são: a adesão e implementação da prescrição eletrônica com devido suporte clínico, inclusão de farmacêuticos nas visitas clínicas, viabilização de contato direto com o farmacêutico durante 24 horas para minimizar as dúvidas relacionadas aos medicamentos e a presença de procedimentos específicos para a prescrição segura ${ }^{19}$.

A Lei Federal no 5.991/1973 que dispõe sobre o controle sanitário do comércio de drogas, medicamentos, insumos farmacêuticos e correlatos, em seu capítulo VI, artigo 35 afirma que a prescrição hospitalar deve ser legível, completa e clara, deve apresentar em geral, o nome, número de registro e leito do usuário, data, nome do medicamento a ser administrado, dosagem, via, frequência e ou horário de administração, duração do tratamento, assinatura legível do médico e número do seu registro profissional, no conselho correspondente, no entanto, mesmo com legislação específica é notável que não é incomum a ausência dessas informações nas prescrições avaliadas ${ }^{15}$

Os resultados referentes à presença de abreviatura, são compatíveis com os dados descritos por Silva et. al., ${ }^{16}$ Gutierrez et. al., ${ }^{20}$ e Oliveira et. al., ${ }^{21}$ onde foram encontradas abreviaturas em 91,5\%, 92,2\% e 91,3\% respectivamente.

A utilização de abreviaturas em prescrições médicas está entre as causas mais mencionadas de erros de medicação, devido ao seu alto potencial de confusão e falhas de comunicação, sendo citada por alguns autores como Rosa et. al., a possibilidade de extinguir seu uso ${ }^{11}$. O protocolo de segurança na prescrição, uso e administração de medicamentos do Ministério da Saúde, condena o uso de abreviaturas nos nomes de medicamentos e propõe que nos ambientes hospitalares, que juguem extremamente necessária a utilização de abreviaturas, seja feita uma lista padronizada dessas e que esta lista seja amplamente difundida entre todos os profissionais do serviço ${ }^{9}$.

A ausência de informação nas prescrições pode dificultar o tratamento e acarretar graves consequências à vida do paciente. Ainda assim, esta pesquisa evidenciou que é comum a prática da omissão de informação nas prescrições, seja essa informação referente a identificação do paciente, ao local de internação, ao diagnóstico, ao prescritor responsável e referente ao medicamento.

\section{CONCLUSÕES}

A pesquisa realizada revela que a prescrição do hospital em estudo precisa ser reavaliada em vários aspectos, principalmente no que diz respeito aos medicamentos, a fim de que sejam evitados erros de medicação e que a assistência prestada aos usuários seja de qualidade e cada vez mais segura.

A insuficiência de informações e a falta de clareza nas prescrições podem confundir os profissionais e causar sérios danos aos usuários, sendo assim, é necessário que se crie mecanismos para viabilizar uma prescrição o mais correta possível, atendendo ao que determinam as legislações específicas e o protocolo de segurança na prescrição, uso e administração de medicamentos do Ministério da Saúde.

\section{Fontes de Financiamento}

A pesquisa não recebeu financiamento para a sua realização.

\section{Conflito de Interesses}

Não há conflitos de interesse relacionados à execução do estudo.

\section{Colaboradores}

DBC, LLAM, ALS e RADMS contribuíram com a concepção, planejamento, análise e interpretação dados, realizaram a redação e revisão crítica do conteúdo. Todos os envolvidos aprovam a versão final a ser publicada. 


\section{Agradecimentos}

Ao Hospital Universitário Lauro Wanderley e a Universidade Federal da Paraíba.

\section{REFERÊNCIAS}

1. Storpirtis S, Mori ALPM, Yochiy A, et al. Ciências Farmacêuticas. Farmácia Clínica e Atenção Farmacêutica. Rio de Janeiro: Guanabara Koogan; 2008.251

2. National Coordinating Council for Medication Error Reporting and Prevention (NCC MERP). The Council: Moving into the Second Decade "Developing Recommendations and Offering Tools", 2000. [acessado 2015 abril 27, 8h50min]. Disponível em: http://www. nccmerp.org/pdf/fifteen_Year_report.pdf

3. Silva BK, Silva JS, Globo AFF, et al. Erros de Medicação: Condutas e propostas de prevenção na perspectiva da equipe de enfermagem. Revista Eletrônica de Enfermagem, 2007, 9(3):712-723.

4. Dean B, Barber N, Schachter V. What is prescribing error? Health Care. Oxford, 2000, 9: 232-237.

5. Cassiani SHB. A segurança do paciente e o paradoxo no uso de medicamentos. Revista Brasileira de Enfermagem, 2005, 58(1): 95 99.

6. Lopes LN, Garcia KP, Dias LG, et al. Qualidade das prescrições médicas em um Centro de Saúde Escola da Amazônia Brasileira. Revista Sociedade Brasileira Clínica Médica, 2014, 12(2): 135-139.

7. Zangwil AB, Bolinger AM, Kamei RK. Reducing prescribing errors through a quiz program formedical residents. Arn. J. Health Syst Pharm, 2000, 30(2):1396-1397.

8. Cassiani SH, Freire CC, Gimenes FRA. A prescrição Médica Eletrônica em um Hospital Universitário: Falhas na redação e opinião dos usuários. Revista Escola de Enfermagem USP, 2003, 37(4): 51-60.

9. Brasil. Protocolo da Segurança na Prescrição, Uso e Administração de Medicamentos. Ministério da Saúde; Fundação Oswaldo Cruz; Agência Nacional de Vigilância Sanitária. Ministério da Saúde, Brasília, 2013.

10. Araújo PTB, Uchôa SAC. Avaliação da qualidade da prescrição de medicamentos de um hospital de ensino. Revista Ciência \& Saúde Coletiva, 2011, 16 (1): 1107-1114.

11. Rosa MB, Perini E, Anacleto TA, et al. Erros na prescrição hospitalar de medicamentos potencialmente perigosos. Revista de Saúde Pública, 2009, 43(3): 490-498.

12. Organização Mundial de Saúde (OMS). Como investigar el uso de medicamentos en los servicios de salud. Indicadores seleccionados del uso de medicamentos. Ginebra, 1993.

13. João WSJ. Reflexões sobre o Uso Racional de Medicamentos. Revista Pharmacia Brasileira, 2010, 78. Disponível em: http://www.cff.org. br/sistemas/geral/revista/pdf/128/015a016_artigo_dr_walter. pdf [acesso em 2015 abril 28].

14. Bozoli LFB. Análise de prescrições para tratamento de câncer de mama em hospital universitário [monografia]. Ribeirão Preto (SP): Universidade de São Paulo; 2013.

15. Brasil. Lei no 5.591, de 17 de dezembro de 1973. Dispõe sobre o controle sanitário do comércio de drogas, medicamentos, insumos farmacêuticos e correlatos e dá outras providências. Brasília. [acesso em 2015 abril 28,15h30min]. Disponível em: http://www.planalto. gov.br/ccivil_03/leis/L5991.htm

16. Silva BK, Silva JS, Globo AFF, et al. Erros de Medicação: Condutas e propostas de prevenção na perspectiva da equipe de enfermagem. Revista Eletrônica de Enfermagem, 2014, 9(3):712-723.

17. Silvério MS, Leite ICG. Qualidade das prescrições em município de Minas Gerais: Uma abordagem farmacoepidemiológica. Revista Associação Médica Brasileira, 2010, 56: 675-680.

18. Aldrigues RFT, Correr CJ, Melchiors AC, et al. Análise da completude de prescrições médicas em uma farmácia comunitária de Fazenda Rio Grande - Paraná (Brasil). Acta Farm Bonaer, 2006, 25: 454-459.

19. Aspden P. Committee on identifying and preventing medication errors.preventing medication errors. institute of medicine of the national academies.Washington. The National Academies Press, 2007: 544.

20. Gutiérrez E, Mogni A, Beron A, et al. Errores de medicación em niños hospitalizados. Archivos de Pediátria del Uruguai, 2011, 82(3). 